\title{
A Note on the Jacobian Conjecture
}

\author{
Zhi-Li Zhang
}

February 1990

\begin{abstract}
We extend a corollary in [2], yielding a sufficient and necessary condition for a polynomial map to have an inverse of the simplest form, and give a surprisingly simple proof for the Jacobian Conjecture in two variables of the case $f_{i}=x_{i}-h_{i}$, where $h_{i}$ is homogeneous of degree $\geq 2, i=1,2$.
\end{abstract}

\section{The Jacobian conjecture}

Let $k$ be a fiels of characteristic 0 , and let $f=\left(f_{1}, \ldots, f_{n}\right)$ be a polynomial map from $k^{n}$ to $k^{n}, f_{i} \in k\left[x_{1}, \ldots, x_{n}\right], 1 \leq i \leq n$.

The Jacobian matrix for $f$ is:

$$
J(f)=\left[\frac{\partial f_{i}}{\partial x_{j}}\right], \quad j(f)=\operatorname{det} J(f)
$$

The Jacobian Conjecture states that if $J(f)$ is invertible, i.e. $j(f)$ is a nonzero constant in $k$, then $f$ has a polynomial inverse.

Although it is trivially true when $n=1$, the Jacobian Conjecture has not been generally resolved even when $n=2$. Only in some special cases has it been proved true([1]):

1. if the degrees of $f_{1}$ and $f_{2}$ do not exceed 100 (Moh).

2. if one of the degrees is of the form $p q$ where $p$ (resp. $q$ ) is 1 or a prime (Abhyankar and Moh, Nakai and Baba). 
3. if one of the degrees is 4 (Nakai and Babai).

4. if the larger of the two degrees is $2 p$ for some odd prime $p$ (Nakai and Baba).

In section 3 , we give a surprisingly simple proof for the case $f_{i}=x_{i}-h_{i}$, where $h_{i}$ is homogeneous of degree $\geq 2, i=1,2$ by using a corollary in [2]. Unfortunately, this simple proof only works for $n=2$.

In the general $n$-variable case, Wang ([1]) proved the Jacobian Conjecture is true if all $f_{i}$ 's have degree 2 . Wright, et al ([2]) reduced the problem to the case where the degree of each $f_{i}$ is at most 3 at the cost of introducing extra variables.

In section 2, we give a weaker condition for the aforementioned corollary in [2] and prove under that condition the converse holds, too. This yields a sufficient and necessary condition for a polynomial map to have an inverse of the simplest form.

\section{The Simplest Inverse}

Without loss of generality $([2])$, we assume $f_{i}$ has the canonical form $f_{i}(\underline{x})=$ $x_{i}-h_{i}(\underline{x})$, where $h_{i}$ has no constant or linear parts. Then, the Jacobian matrix for $f$ is:

$$
J(f)=\left[\frac{\partial f_{i}}{\partial x_{j}}\right]=I-\left[\frac{\partial h_{i}}{\partial x_{j}}\right], j(f)=\operatorname{det} J(f)
$$

Furthermore, if $J(f)$ is invertible, we assume $j(f)=1$. Observe that if $h(\underline{x})$ is homogeneous, then $J(h)$ is a nilpotent matrix.

The following lemma describes the sufficient and necessary condition for the inverse of $f(x)$ to have the simplest form $g(\underline{x})=\underline{x}+h(\underline{x})$, when $h(\underline{x})$ is homogeneous. From Abhyankar Inversion Formula in [2], we see for $f(\underline{x})$ of the canonical form with $h(\underline{x}) \neq 0$ and homogeneous, the inverse of $f(\underline{x})$ contains $\underline{x}+h(\underline{x})$ as the first two lower degree parts, this justifies our usage of the word simplest.

Lemma 1 Let $f(\underline{x})=\underline{x}-h(\underline{x}), h(\underline{x})$ homogeneous of degree $d \geq 2$, and 
assume $j(f)=1$, then $f$ is invertible with inverse $g(\underline{x})=\underline{x}+h(\underline{x})$ iff $J(h(\underline{x})) \cdot h(\underline{x})=0$ i.e. $J(h(\underline{x}))^{2} \underline{x}=0$.

Proof: if part: Recall Taylor Expansion Formula on vector space of functions:

$$
f(\underline{x}+\triangle \underline{x})=f(\underline{x})+\nabla f(\underline{x}) \cdot \triangle \underline{x}+\ldots+\nabla^{t} f(\underline{x})(\triangle \underline{x})^{t}+\cdots
$$

where $\nabla$ is the differential operator, $\nabla^{t} f(\underline{x})$ is a $t$-dimensional matrix.

$$
\left.\nabla^{t} f(\underline{x})(\triangle \underline{x})\right)^{t}=\left(\ldots(\nabla^{t} f(\underline{x}) \underbrace{\triangle \underline{x}) \ldots) \triangle \underline{x}}_{t}\right.
$$

Apply the above formula to $h(\underline{x}-h(\underline{x})): \quad(\triangle \underline{x}=-h(\underline{x}))$

$$
h(\underline{x}-h(\underline{x}))=h(\underline{x})-\nabla h(\underline{x}) \cdot h(\underline{x})+\cdots+(-1)^{t} \nabla^{t} h(\underline{x}) \cdot h^{t}(\underline{x})+\cdots
$$

By inducing on $t$, we prove that $0=J(h(\underline{x}) h(\underline{x}))=\nabla h(\underline{x}) \cdot h(\underline{x})$ implies

$$
\nabla^{t} h(\underline{x}) \cdot h^{t}(\underline{x})=0 \text {, for all } t \geq 1 \text {, thus } h(\underline{x}-h(\underline{x}))=h(\underline{x}) .
$$

This yields $h(\underline{x})=h(g)$, but $g(\underline{x})=\underline{x}+h(g)$, therefore $g(\underline{x})=\underline{x}+h(\underline{x})$.

Now assume $\nabla^{t-1} h(\underline{x}) \cdot h^{t-1}(\underline{x})=0$.

Apply $\nabla$ once more, by chain rule, we have

$$
\begin{aligned}
0= & \nabla\left(\nabla^{t-1} h(\underline{x}) \cdot h^{t-1}(\underline{x})\right)=\nabla^{t} h(\underline{x}) \cdot h^{t-1}(\underline{x})+ \\
& +\nabla^{t-1} h(\underline{x})\left(\sum_{\substack{i+j=t-2 \\
0 \leq i, j \leq t-2}} h^{i} \nabla h(\underline{x}) \cdot h^{j}(\underline{x})\right)
\end{aligned}
$$

Multiply $h(\underline{x})$ to the right, and notice that $\nabla h(\underline{x}) \cdot h(\underline{x})=0$.

We conclude $\nabla^{t} h(\underline{x}) \cdot h^{t}(\underline{x})=0$, this completes the induction.

only if part: As $g(\underline{x})=\underline{x}+h(g)$, we have $h(\underline{x})=h(g)=h(x+h(\underline{x}))$. Apply Taylor Expansion to $h(x+h(\underline{x}))$ with $\triangle \underline{x}=h(\underline{x})$. As $h(\underline{x})$ is homogeneous of degree $d \geq 2$, and $\nabla^{t} h(\underline{x}) \cdot h^{t}(\underline{x})$ has degree 
$(d-t)+t d$, for $1 \leq t \leq d$, whereas

$\nabla^{t} h(\underline{x})=0$ for $t>d$, it follows that

$$
\nabla^{t} h(\underline{x}) \cdot h^{t}(\underline{x})=0, \text { for all } t \geq 1
$$

In particular, $\nabla h(\underline{x}) \cdot h(\underline{x})=J(h) h=0$.

Remark If $h$ is homogeneous of degree $d \geq 2$, by Euler's Theorem for homogeneous functions, $h_{i}=\frac{1}{d}\left(\sum_{j=1}^{n} \frac{\partial h_{i}}{\partial x_{j}} x_{j}\right)$, hence $h(\underline{x})=\frac{1}{d} J(h(\underline{x})) \underline{x}$. It is clear that $J(h)^{2}=0$ implies that $J(h) h=\frac{1}{d} J(h)^{2} \underline{x}=0$. On the other hand, for a general matrix $\mathrm{M}$ over $k[\underline{x}], M^{2} \underline{x}=0$ for all $\underline{x} \in k^{n}$ does not necessarily imply $M^{2}=0$.

For example, let

$$
M=\left[\begin{array}{ll}
x_{2} & -x_{1} \\
x_{2} & -x_{1}
\end{array}\right]
$$

we have $M \underline{x}=0$, hence $M^{2} \underline{x}=0$, but $M^{2} \neq 0$.

Therefore, in genreal, the condition of Lemma 1 is slightly weaker than the condition of Corollary 5.4 in [2], an under this weaker condition the converse holds, too.

However, as the matrix in question is the Jacobian matrix $J(h(\underline{x}))$ for homogeneous functions $h(\underline{x})$, it could happen that $J(h(\underline{x}))^{2}=0$ is equivalent to $J(h(\underline{x}))^{2}=0$ in this specific setting. This is the case when $n=2$, as $j(h)=1$ implies $J(h)^{2}=0$ (see the proof of Theorem 2). When $n=3$, $J(h)^{2}=0$ impies that the rank of $J(h)$ is 1 , or the compound matrix of $J(h)$ is zero, whereas $J(h)^{2} \underline{x}=J(h) h=0$ gives no hint of the rank of $J(h)$. For $n>3$, no simple things can be said. We believe the two conditions are not equivalent when $n \geq 3$.

As for homogeneous $h(\underline{x})$, we know $J(h(\underline{x}))$ is nilpotent. Lemma 1 points out a simple relation between the nilpotency of $J(h)$ ( or rather, a modified condition on the nilpotency of $J(h)$ ) and the form the inverse of $f(\underline{x})=$ $\underline{x}-h(\underline{x})$ may take. One might like to further investigate this relationship and ask:

Does $J(h(\underline{x}))^{k}=0$ or $J(h(\underline{x}))^{k} \underline{x}=0$ or other similar expressions give a sufficient and/or necessary condition for the inverse 
of $f(\underline{x})=\underline{x}-h(\underline{x})$ to take some simple form, e.g. as might be suggested by the Abhyankar Inversion Formula?

The answer seems to be negative.

\section{The Jacobian Conjecture In Two Variables}

In this section, we prove that when $n=2$ and $h(\underline{x})$ homogeneous, $f(\underline{x})=$ $\underline{x}-h(\underline{x})$ is invertible, with the simplest inverse $g(\underline{x})=\underline{x}+h(\underline{x})$ by showing $J(h)^{2}=0$. Homogeneity of $h(\underline{x})$ plays the key role in the proof.

Theorem 2 For $f=\left(f_{1}, f_{2}\right), f_{i}=x_{i}-h_{i}$, where $h_{i}$ is homogeneous of degree $\geq 2, i=1,2$. Assume $j(f)=1$, then $f$ is invertible.

Proof: As $h_{1}, h_{2}$ are homogeneous,

$$
1=j(f)=1-\frac{\partial h_{1}}{\partial x_{1}}-\frac{\partial h_{2}}{\partial x_{2}}-\frac{\partial h_{1}}{\partial x_{2}} \frac{\partial h_{2}}{\partial x_{1}}+\frac{\partial h_{1}}{\partial x_{1}} \frac{\partial h_{2}}{\partial x_{2}}
$$

implies

$$
\frac{\partial h_{1}}{\partial x_{1}}+\frac{\partial h_{2}}{\partial x_{2}}=0, \quad \frac{\partial h_{1}}{\partial x_{1}} \frac{\partial h_{2}}{\partial x_{2}}=\frac{\partial h_{1}}{\partial x_{2}} \frac{\partial h_{2}}{\partial x_{1}}
$$

Therefore

$$
\left(\frac{\partial h_{1}}{\partial x_{1}}\right)^{2}+\frac{\partial h_{1}}{\partial x_{2}} \frac{\partial h_{2}}{\partial x_{1}}=\left(\frac{\partial h_{1}}{\partial x_{1}}\right)^{2}+\frac{\partial h_{1}}{\partial x_{1}} \frac{\partial h_{2}}{\partial x_{2}}=0
$$

Similarly,

$\frac{\partial h_{2}}{\partial x_{1}} \frac{\partial h_{1}}{\partial x_{1}}+\frac{\partial h_{2}}{\partial x_{2}} \frac{\partial h_{2}}{\partial x_{1}}=0, \quad \frac{\partial h_{1}}{\partial x_{1}} \frac{\partial h_{1}}{\partial x_{2}}+\frac{\partial h_{1}}{\partial x_{2}} \frac{\partial h_{2}}{\partial x_{2}}=0, \quad \frac{\partial h_{2}}{\partial x_{1}} \frac{\partial h_{1}}{\partial x_{2}}+\left(\frac{\partial h_{2}}{\partial x_{2}}\right)^{2}=0$

Thus, we have shown $J(h)^{2}=0$. By Lemma $1, f$ is invertible. 


\section{References}

[1] S. Wang A Jacobian criterion for separability J.Algebra 65 (1980), 453494.

[2] H. Bass, E. Connell, and D. Wright The Jacobian Conjucture: Reduction of Degree and Formal Expansion of the Inverse Bull. of the Amer. Math. Soc. Vol 7, No 2 (1982), 287-330. 